\title{
Neurocognitive Changes in Tertiary Neurosyphilis: A Retrospective Chart Review
}

\author{
Philippe Beauchemin, Robert Laforce Jr
}

\begin{abstract}
Context: Since the beginning of the new millennium, prevalence of syphilis has re-increased and is once again, a major public health problem. Neurosyphilis is the extension of syphilitic infection to the nervous system. It is considered by many as a cause of reversible dementia, when treated early. However, scarce data exist on the evolution of cognitive and behavioral impairments in patients affected by tertiary neurosyphilis. Objectives: The aim of this study was to explore the cognitive and behavioral changes in a cohort of patients diagnosed with neurosyphilis. Design: A retrospective study based on systematized chart review between 2000 and 2012 in a large neurological tertiary care facility. Outcome measure: Clinical evaluations by treating physicians. Results: Eighteen patients were identified with tertiary neurosyphilis. Out of this group, only two had systematic neuropsychological follow-up despite physician reports of significant and persistent cognitive and psychiatric changes. For these two cases, only slight improvements were noted in memory and executive skills while improvements in attention were marked. None of our patients had previous psychiatric history yet a large proportion developed symptoms after the infection. Conclusion: Although neurosyphilis is traditionally considered a reversible form of dementia, we found limited support for this claim in our two patients with close follow-up. Quality data on the cognitive and psychiatric changes in the rest of our cohort was dramatically lacking, and this could not be explained by absence of symptoms at presentation. Given the recrudescence of syphilis, we propose a systematic approach to the evaluation and follow-up of this disorder.
\end{abstract}

RÉSUMÉ: Changements neurocognitifs dans la neurosyphilis tertiaire : une étude rétrospective. Contexte : Depuis le début du nouveau millénaire, la prévalence de la syphilis a augmenté de nouveau et elle est devenue encore une fois un problème de santé publique majeur. La neurosyphilis est une extension de l'infection syphilitique au système nerveux. Elle est considérée par plusieurs comme une cause réversible de démence lorsque traitée tôt. Cependant, il existe peu de données sur l'évolution des déficits cognitifs et comportementaux chez les patients atteints de neurosyphilis tertiaire. Objectifs : Le but de cette étude était d'explorer les changements cognitifs et comportementaux chez une cohorte de patients avec un diagnostic de neurosyphilis. Devis d'étude : Nous avons effectué une étude rétrospective systématique des dossiers de patients traités entre 2000 et 2012 dans un hôpital de soins neurologiques tertiaires. Résultats : Dix-huit patients qui présentaient une neurosyphilis tertiaire ont été identifiés. Parmi ces patients, seulement deux ont fait l'objet d'un suivi neuropsychologique systématique malgré le fait que des changements cognitifs et psychiatriques persistants importants ont été noté par le médecin chez la plupart. Dans ces deux cas, des améliorations mineures de la mémoire de travail et des fonctions exécutives ont été notées alors que l'attention s'est améliorée de façon importante. Aucun de nos patients n'avait d'antécédent psychiatrique bien qu'une grande proportion présentait des symptômes après l'infection. Conclusion : Bien que la neurosyphilis soit traditionnellement considérée comme une forme réversible de démence, nous avons constaté peu d'éléments à cet effet chez les deux patients ayant bénéficié d'un suivi serré. Dans le reste de notre cohorte, peu de données de qualité sur les changements cognitifs et psychiatriques étaient disponibles et ceci ne pouvait s'expliquer par une absence de symptômes initiaux.

Can J Neurol Sci. 2014; 41: 452-458

Syphilis is a chronic infection caused by the spirochete Treponema pallidum. It is most commonly transmitted through sexual contact. ${ }^{1}$ Recently, it has become a major public health problem once again. Through the mid 80 's, bisexual and homosexual men comprised almost $50 \%$ of cases. It was also prevalent in the HIV-positive population. ${ }^{2}$ After a gradual disappearance during the 90 's, overall case rates began to increase worldwide since $2000 .^{3}$ For example, in the province of Quebec, Canada (population of eight million inhabitants), recent epidemiological surveys indicate an increase from 3 cases in 1998 to 671 cases in $2012 .^{4}$
Primary syphilis presents as a painless chancre, which heals within two to three weeks. Secondary syphilis presents in $25 \%$ of untreated patients within weeks to months of primary

From the Clinique Interdisciplinaire de Mémoire (PB, RLJ), Département des Sciences Neurologiques, CHU de Québec; Faculté de Médecine (RLJ), Université Laval, Québec, Canada.

Received August 8, 2013. Final Revisions Submitted February 5, 2014. Correspondence to: Robert Laforce Jr, Département des Sciences Neurologiques, CHU de Québec, 1401, 18ième rue, Québec, G1J 1Z4, Canada.

Email: robert.laforce@fmed.ulaval.ca. 
infection. ${ }^{5}$ Common findings of secondary syphilis include rash of the palms and soles, fever, lymphadenopathy and central nervous system (CNS) changes. Forty percent of patients with secondary syphilis have CNS symptoms of visual disturbances, hearing loss, tinnitus and facial weakness. ${ }^{6}$ After a latency period, $25 \%$ of untreated patients will develop tertiary syphilis. ${ }^{6}$ Tertiary syphilis occurs between 1 to 30 years after primary infection. ${ }^{5}$ At this stage, the disease is slowly progressive and presents as meningovascular, parenchymatous, cardiovascular or in gummatous forms. ${ }^{7}$

Neurosyphilis literally means syphilitic infection of the CNS, but it is often incorrectly named tertiary syphilis. ${ }^{8}$ Indeed, neurosyphilis can occur at any time over the course of syphilis, even in its primary stage. ${ }^{8}$ The term neuroinvasion is used when the organism disseminates to cerebrospinal fluid (CSF) and meninges. ${ }^{9}$ Central nervous system pathologic findings include meningovascular and parenchymatous neurosyphilis. Meningovascular neurosyphilis usually occurs five to ten years after initial infection and produces classic findings ranging from progressive neurologic deficits to aphasia and seizures. Parenchymatous neurosyphilis develops 10 to 20 years after initial infection. It includes tabes dorsalis and general paresis. Early paresis is thought to be associated with subtle deterioration of cognition with poor concentration, irritability and loss of higher cortical functions. Associated psychiatric symptoms described by previous authors include acute psychosis, paranoid delusions, personality changes, affective disorders and cognitive impairments. ${ }^{10}$

Although traditionally considered as a reversible cause of dementia when treated early, scarce data exist on the evolution of cognitive and behavioral impairments in patients affected by tertiary neurosyphilis. Moreover, the sequelae associated with these neurocognitive changes have rarely been addressed. The aim of this study was to explore the cognitive and behavioral changes in a cohort of patients diagnosed with neurosyphilis.

\section{Methods}

We performed a retrospective chart review of patients with a clinical diagnosis of tertiary neurosyphilis, hospitalized in a large neurological tertiary care facility between 2000 and 2012. All cases fulfilled Centers for Disease Control (CDC) criteria ${ }^{11}$ : 'Confirmed neurosyphilis' (any syphilitic stage and a reactive CSF-venereal disease research laboratory (VDRL)) or 'Presumptive neurosyphilis' (any syphilitic stage, a non-reactive CSF-VDRL, elevated CSF protein or white blood count (WBC) in the absence of other known causes, and clinical symptoms and signs consistent with neurosyphilis without other known causes for these clinical abnormalities). If diagnosis was remote to study period, patients were included when ample information was available to confirm diagnosis. All patients were treated by Infectiologists with antibiotics using standard regimens. Exclusion criteria were: a primary or a secondary syphilis without evidence of CNS involvement. No individuals were excluded based on pre-existing cognitive impairment or psychiatric conditions.

Each patient's chart with a coded diagnosis of syphilis was reviewed using a systematic data collection form focusing on demographics, neurological signs, cognitive and psychiatric evaluations. The entire patient's charts were studied. Physicians' notes and discharge summary were all studied along with consultations from various specialists (Infectiology, Psychiatry, Internal Medicine, Geriatrics). Neurological signs were extracted only from Neurology consultations, however. Cognitive assessments (brief screening measures and/or detailed neuropsychological evaluations) were extracted independently of the source. Data from blood analysis, brain imaging and CSF analysis were taken directly from chart reports.

Descriptive statistical techniques (means, percentages) were used to analyze the results. For the two patients' charts for which we had access to detailed neuropsychological assessments, data were summarized according to five main cognitive domains: 1) Attention (Digit Span of the Wechsler Adult Intelligence Scale III (WAIS-III), Trail Making Test - A), 2) Memory (California Verbal Learning Test - recall, Rey Complex Figure Test (RCFT) - recall, Bushke Selective Reminding Test), 3) Executive (DKefs, Controlled Oral Word Association Test, RCFT copy), 4) Language (Boston Diagnostic Assessment Examination naming), and 5) General intellectual abilities (WAIS-III).

This study was approved by our local Research Ethics Board.

\section{RESULTS}

Thirty nine medical records were initially reviewed. Twentyone patients were excluded, that is seventeen who had isolated primary or secondary syphilis without evidence of CNS involvement, and four with lack of reliable information to meet CDC criteria. A final sample of 18 patient records was therefore included in this study. Although the total number of charts that had complete records according to our data collection form was $61 \%(11 / 18)$, in depth analysis revealed dramatic lack of quality data and formal comprehensive cognitive and psychiatric evaluations.

Demographics are shown in Table 1. Two thirds of patients are male with a mean age of 57-years-old. This is congruent with the age range of tertiary syphilis, which occurs after a long incubation period. Out of five patients tested for HIV, only one was positive. Although Argyll-Robertson pupils and deafness are

Table 1: Demographics

\begin{tabular}{lll} 
Characteristic & Category & \% of patient (number $\mathbf{1 8})$ \\
Gender (male) & & $67 \%(12)$ \\
\hline Age: mean (range) & & $57(31-83)$ \\
\hline \multirow{2}{*}{ Ethnic origin } & Caucasians & $83 \%(15)$ \\
& Blacks & $11 \%(2)$ \\
& Asians & $6 \%(1)$ \\
\hline HIV positive & & $1 / 5$ \\
\hline & Seizures & $28 \%(5)$ \\
& Hyperreflexia & $22 \%(4)$ \\
Neurological & Ophthalmoparesis & $17 \%(3)$ \\
impairments & Stroke* & $17 \%(3)$ \\
& Argyll-Robertson & $11 \%(2)$ \\
& pupils & \\
& Deafness & $11 \%(2)$
\end{tabular}

*All strokes were radiological. HIV: Human immunumumuny vau. 
traditionally considered part of the neurological findings in neurosyphilis, it was noted in only $11 \%$ of our patients. Diffuse hyperreflexia, a non-specific finding, was found in $22 \%$ of patients. Stroke (radiological), a marker of the meningovascular form, was found in $17 \%$ of patients.

Cognitive changes were noticed in the vast majority of patients $(83 \%)$, with memory impairment being the most frequent $(44 \%)$ followed by executive dysfunctions $(33 \%)$ (Table 2). Visuospatial impairments were much less commonly reported. The average MMSE, a widely used cognitive screening test done at baseline, was 23.6/30, with a range between 16 and 29 . Additional random cognitive testing was conducted in only 8/18 patients. Formal comprehensive and longitudinal neuropsychological evaluation and follow-up was only available in $2 / 18$ charts reviewed. Figure 1 illustrates the neuropsychological profiles at baseline and six months post-treatment in these two cases. The first of these two cases (A) is a 74-yearold male with a high school education who presented with dysarthria and confusion. The second case (B) is a 59-year-old female with a high-school education who presented with diplopia, apathy, confusion and marked memory loss. Both had average IQ. Language was well preserved. Interestingly, only slight improvements were noted in memory and executive skills, while attention tended to improve over time. Figure 2 provides a graphic example of the improvements noted in executive skills in
Table 2: Cognitive and behavioral changes

\begin{tabular}{|c|c|c|}
\hline Changes & Domains & $\%$ of patients (n/18) \\
\hline \multirow{8}{*}{ Cognitive } & Total§ & $83 \%(15)$ \\
\hline & Memory & $44 \%(8)$ \\
\hline & Executive & $33 \%(6)$ \\
\hline & Judgment & $28 \%(5)$ \\
\hline & Attention & $17 \%(3)$ \\
\hline & Perseveration & $17 \%(3)$ \\
\hline & Language & $17 \%(3)$ \\
\hline & Visuospatial & $11 \%(2)$ \\
\hline \multirow{6}{*}{ Psychiatric } & Total $\S \S$ & $89 \%(16)$ \\
\hline & Delusions* & $61 \%(11)$ \\
\hline & Hallucinations & $22 \%(4)$ \\
\hline & Depression & $17 \%(3)$ \\
\hline & Anxiety & $11 \%(2)$ \\
\hline & Pathological gambling & $11 \%(2)$ \\
\hline
\end{tabular}

Cognitive and behavioral changes were calculated based on history, bedside and/or brief cognitive testing and/or formal comprehensive neuropsychological assessment. 'Total§' means the total number of patients' charts who cited at least one cognitive change. 'Total\$§' means the total number of patients' charts who cited at least one psychiatric change. *Delusions include persecutory $(n=4)$, grandiose $(n=1)$ and non-specified $(\mathrm{n}=6)$.
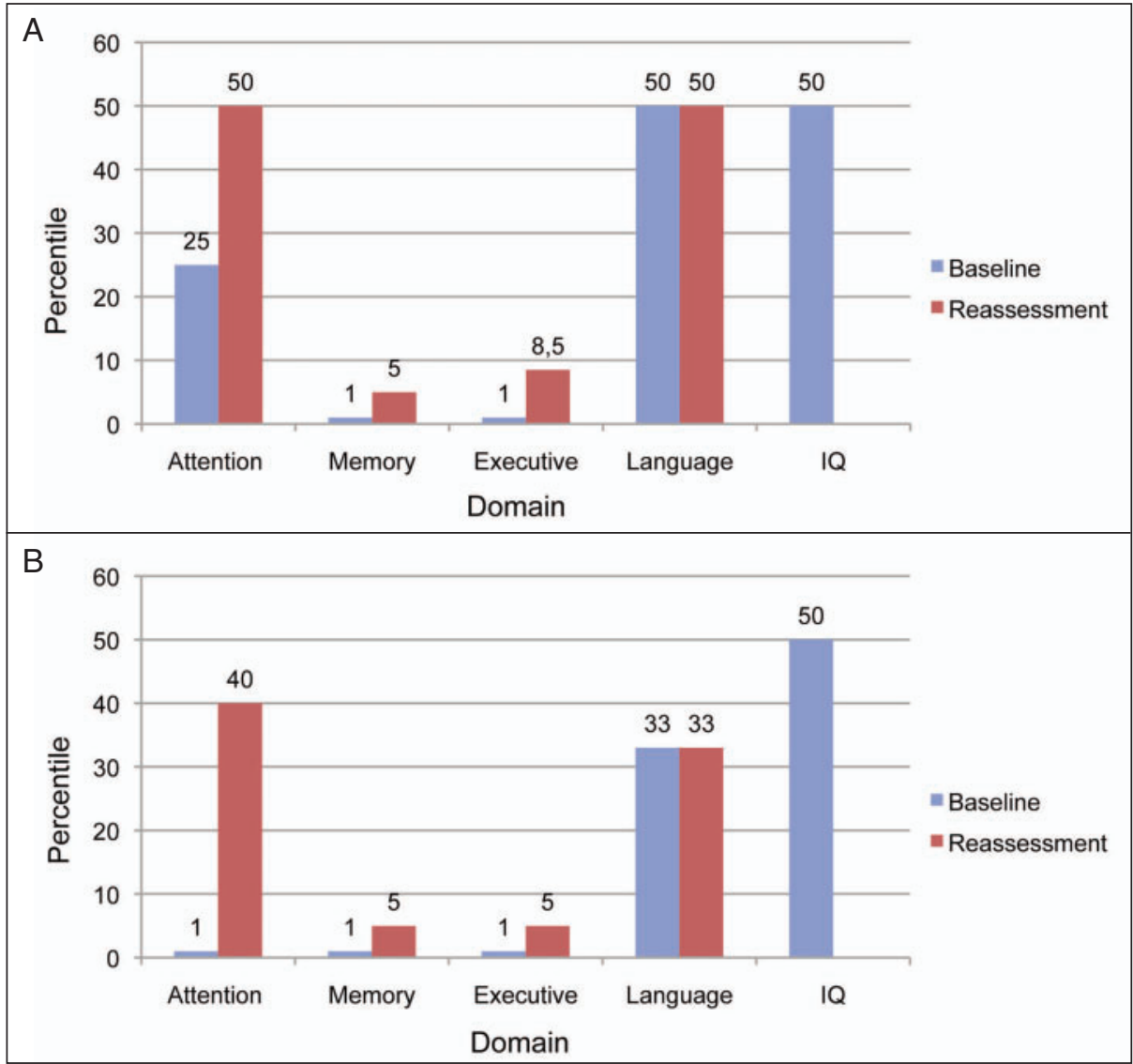

Figure 1: Longitudinal neuropsychological profile over a six-month period in A) a 74 year-old male with a high-school education who presented with dysarthria and confusion in addition to cognitive changes. Follow-up neuropsychological reassessment revealed improvement in attention while memory and executive functions also improved but to a lesser extent. B) A similar profile of improvement in attention with limited improvement in memory and executive functions was observed in this 59 year-old female with a high-school education who presented with diplopia, confusion and marked memory loss. 


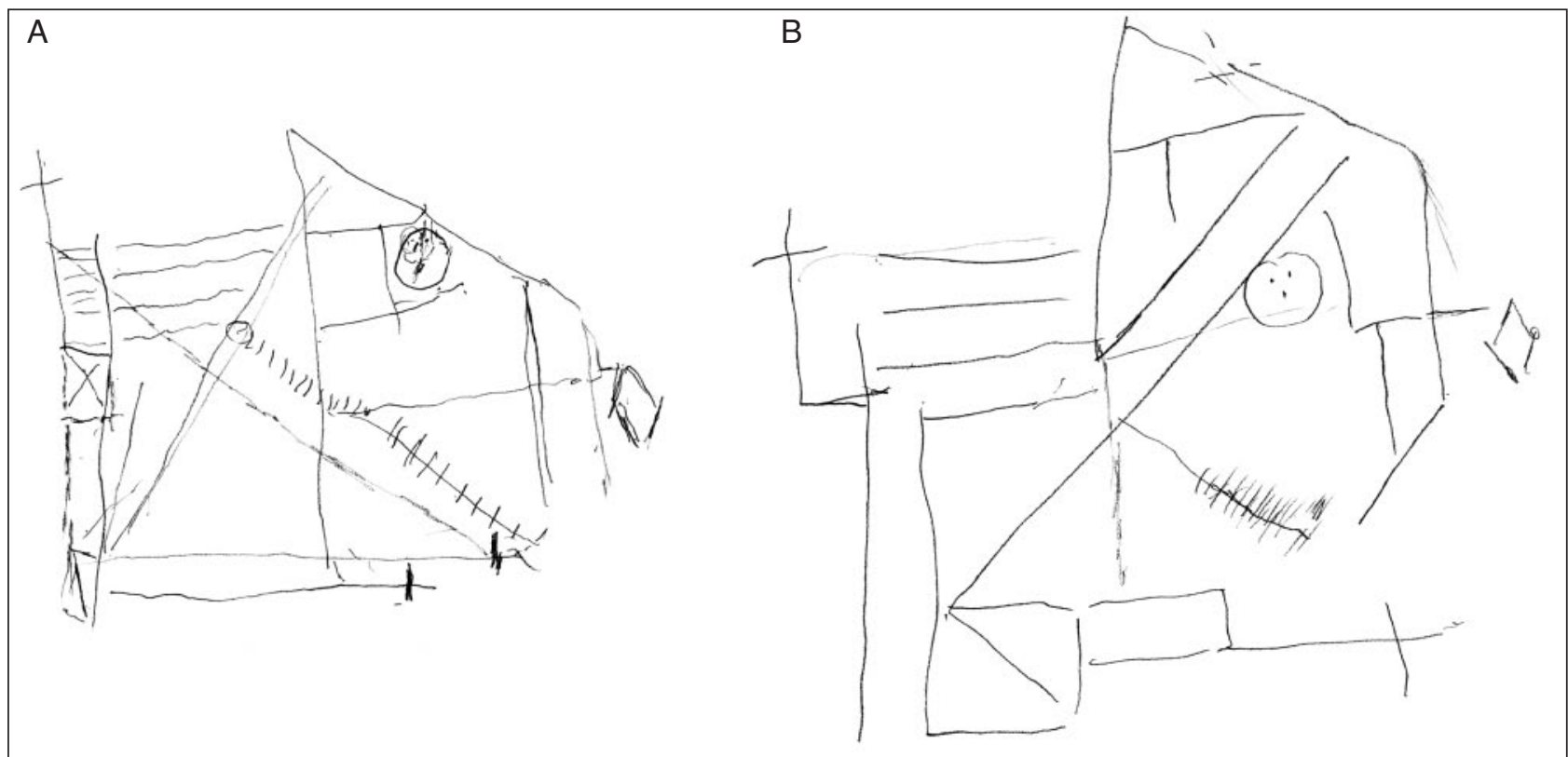

Figure 2: Copy of the Rey Complex Figure performed A) at baseline by a 59-year-old female patient. This mainly shows disorganized constructional skills as well as perseverations in drawing. The patient proceeded by juxtaposing details of the original image. B) At six-months post-treatment, marked improvements in overall planning and constructional abilities are seen. Moreover, there are no remaining perseverative features. Despite these improvements, however, performance is well below expected premorbid level of functioning.

a 59-year-old female patient on the Rey Complex Figure test, both at baseline and six months post-treatment.

Psychiatric features were found in an even greater proportion than cognitive changes in our sample (89\%) (see Table 2). It is important to note that none of our patients were known for a psychiatric illness prior to diagnosis. A large proportion showed delusions (61\%), especially of the persecutory type. This was followed by hallucinations (mostly auditory) in $22 \%$ of cases. Depression and anxiety were less frequent (17\% and $11 \%$, respectively). Pathological gambling was only found in two charts.

Laboratory results are presented in Table 3. Every patient had a positive Treponema pallidum particle agglutination (TP-PA) and a positive non-treponemal rapid plasma reagin (RPR)

Table 3: Laboratory results

\begin{tabular}{lll} 
Tests & Results & \% of patients (n/18) \\
Brain MRI & Normal & $17 \%(3)$ \\
& Abnormal & $61 \%(11)$ \\
& No brain MRI & $22 \%(4)$ \\
\hline & Non-specific findings & $71 \%(10)$ \\
MRI findings & Bilateral mesio-temporal hypersignal & $21 \%(3)$ \\
\hline TP-PA & Syphilitic gumma & $14 \%(2)$ \\
\hline RPR (range) & Positive & $100 \%(18)$ \\
\hline CSF analysis & Positive $(1 / 1-1 / 512)$ & $100 \%(18)$ \\
White Blood Cells count: mean (range) x $10^{\mathbf{6}}$ & $45(0-124)$ & \\
Proteins: mean (range) g/L & $0.57(0.22-1.04)$ & $44 \%(8)$ \\
OCB: mean (range) & $1.2(0-6)$ & \\
Positive VDRL & & \\
VDRL titer & $1 / 8-1 / 128$ & \\
\hline
\end{tabular}

Only 14/18 patients had an MRI of the brain. Non-specific and other findings: cerebral atrophy $(n=6)$, non-specific white matter disease $(n=4)$, radiological stroke $(n=3)$. Abbreviations: CSF: Cerebrospinal fluid; MRI: Magnetic resonance imaging; OCB: Oligoclonal bands; RPR: Rapid plasma reagin; TP-PA: Treponema pallidum particle agglutination; VDRL: Venereal disease research laboratory. 

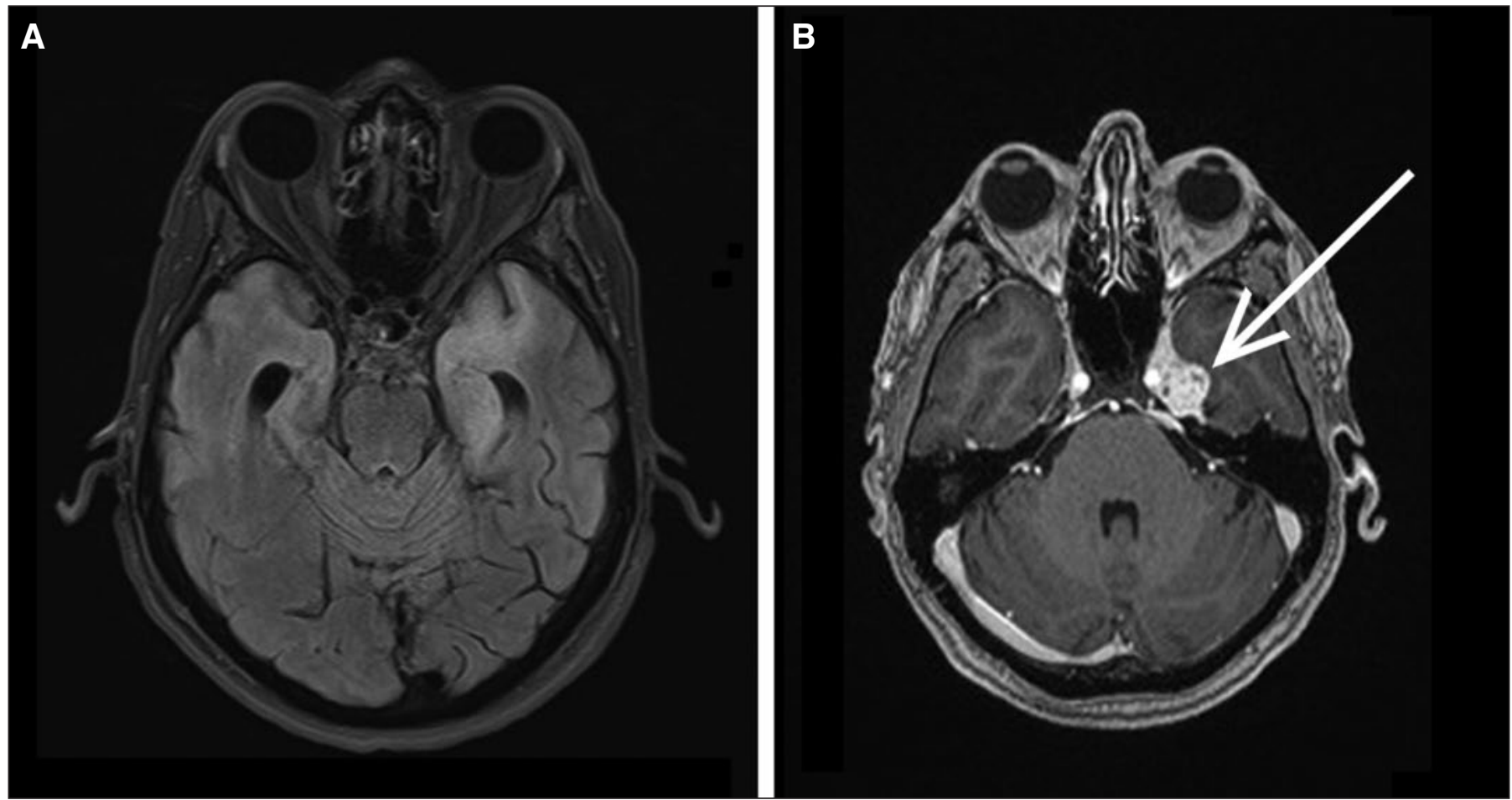

Figure 3: Spectrum of imaging abnormalities associated with neurosyphilis. A) Neurosyphilis mimicking limbic encephalitis with a FLAIR sequence showing bilateral asymmetric $(L>R)$ hyper-intensities in mid-temporal structures. There was also mild diffuse atrophy. This image is from a 59-year-old female patient described earlier in the text. B) Syphilitic gumma in cavernous sinus on T1-gadolinium MRI sequence of a 48 year-old male. An enhancing lesion is found in the left cavernous sinus (white arrow). This lesion substantially diminished in size with penicillin treatment (image not shown).

serology to confirm diagnosis. Less than half $(44 \%)$ of patients had positive CSF-VDRL titers, ranging from $1 / 8$ to $1 / 128$. Cerebrospinal fluid pleocytosis was common, with a mean of 45 cells (range 0 to 124 cells per $\mathrm{mm}^{3} \times 10^{6}$ ), mainly lymphocytes. Oligoclonal bands were positive in seven patients, with a maximum of six bands. Eleven patients had abnormal brain magnetic resonance imaging (MRI). More than half had focal or diffuse cerebral atrophy $(6 / 11)$. Cerebral white matter changes were found in $36 \%(4 / 11)$ of patients. Three patients had abnormal temporal signal mimicking herpes simplex encephalitis. Syphilitic gumma was found in two patients, one in cavernous sinus and another one at the cortico-sub-cortical junction in temporal lobe. Figure 3 illustrates the spectrum of imaging abnormalities associated with neurosyphilis in our cohort.

\section{Discussion}

This retrospective study of a cohort of 18 patients with tertiary neurosyphilis showed very limited quality data in terms of the cognitive and behavioral symptoms associated with the disease over time. Despite physician reports of significant and persistent cognitive changes, very limited cases included detailed assessments. In two well-documented cases that were studied over a six-month period, little improvements were found in memory and executive skills while marked ameliorations were noted in attentional functions. A striking finding was that cases without any previous psychiatric comorbidities appeared to develop significant permanent psychiatric symptoms. Although neurosyphilis is traditionally considered a reversible form of dementia, we found limited support for this claim in our welldocumented cases, and in the literature.

In the early part of the 20th century, psychiatric manifestations of neurosyphilis were common and accounted for 5 to $15 \%$ of first admissions to mental health hospitals. ${ }^{12}$ According to some authors ${ }^{13}$, the diagnosis of neurosyphilis could be differentiated from other known psychoses by characteristic abnormality in eye pupil reflexes, development of muscular reflex abnormalities, seizures, memory impairment (dementia) and other signs of relatively pervasive neurological deterioration. Interestingly, classic signs described earlier in the literature such as Argyll-Robertson pupils, diffuse hyperreflexia or deafness were not frequent in our cohort. However, cognitive changes, especially memory and executive impairments, were common and frequently associated with psychiatric disturbances, such as delusions and hallucinations.

We were quite puzzled by the fact that in our cohort, several patients without any previous psychiatric co-morbidities developed late-onset psychiatric symptoms. Old age can represent a red flag for new-onset psychiatric symptoms. In another cohort $^{12}$, the most frequent clinical symptoms presenting on admission were a wide variety of psychiatric-behavioral symptoms, including emotional problems (92.2\%), personality 
changes (87.9\%), abnormal behavior (84.5\%), delusions $(38.8 \%)$, mostly delusions of persecution, and hallucinations $(12.9 \%)$. Cognitive impairment $(85.3 \%)$, incontinence $(35.3 \%)$, sleep-wake perturbations (25.0\%) and epileptic seizures (16.4\%) were also encountered in that cohort. Palsy, unsteady gait, dysarthria and tremor were observed as well. The neuroanatomical basis of these findings can probably be explained by involvement of medial temporal lobe structures or limbic and frontal circuits involved in the regulation of behavior.

Another key observation from our retrospective effort is the absence of systematic longitudinal follow-up. Even if considered a reversible cause of dementia, limited data exist on the actual reversible nature of cognitive and behavioral changes in tertiary neurosyphilis. There have been reports of both improvement and deterioration in the mental status of such patients after treatment. In one study ${ }^{7}$, it was estimated that neurosyphilis could be arrested in approximately $80 \%$ of cases, producing complete $(33 \%)$ or partial $(47 \%)$ remission of functional impairment. In another study ${ }^{14}$, from 52 patients whose outcome was evaluated, 21 recovered completely, ten had psychiatric disorders, seven had residual seizures, seven had residual hemiparesis, one suffered from tabes dorsalis and six died. Our design did not account for those patients who possibly were lost to follow-up because of significant improvements in their condition.

Patients affected by neurosyphilis frequently maintain deficits in social functioning, personality changes and a generalized dementia. ${ }^{15}$ Irreversible brain damage often occurs before treatment and sometimes progresses even after therapy. ${ }^{15}$ This is specifically what was found in our two patients. In most cases and despite the literature on the subject, formal neuropsychological evaluation at baseline and after treatment was lacking from the charts. Indeed, random cognitive testing was performed in 8 out of 18 patients and a variety of instruments were used. This certainly had an impact on our data which explored the prevalence and evolution of clinical deficits in neurosyphilis.

A number of imaging features reported in the literature were found in our sample. As many as two thirds of patients with neurosyphilis are reported to have a normal CT (computed tomography) scan of the brain, MRI and/or angiography or nonspecific mild-to-moderate cerebral atrophy. Cerebral infarction and white matter lesions are described in nearly $25 \%$ and $20 \%$ respectively. ${ }^{16}$ This is comparable to our findings. Other findings are leptomeningeal enhancement indicative of focal or diffuse meningitis, arteritis, leptomeningeal granulomas, meningoneuritis with cranial neuropathies, orbital gummatous periostitis and periostitis involving the otic capsule. ${ }^{11,17,18}$ One study showed that medial temporal lobe atrophy may be a good prognostic sign of bad evolution in general paresis. ${ }^{19}$ In the medial temporal lobe atrophy group, a personality change or general dementia remained even after the treatment was completed, and outcome in social functioning was poor. The reason why atrophy occurs in the medial temporal lobe is unclear, but it can occur secondarily to dysfunction in diffuse cerebral cortex, as the medial temporal regions have connections with cerebral association areas.

There are several limitations to our study, many of which have already been mentioned earlier in the discussion. Our data remains descriptive and is based on retrospective chart reviews.
Our results are dependent on proper recognition and reporting of key findings by the treating physician. Only $61 \%$ of our charts had complete records according to our data collection form and when explored in greater depth there was a dramatic lack of quality data particularly for formal comprehensive cognitive and psychiatric evaluations, and functional status. Follow-up and testing were heterogeneous both from a medical and neurocognitive perspective. Formal cognitive evaluation by a neuropsychologist was done in only a few patients. These detailed evaluations are useful to draw a complete picture of cognitive decline. Many patients were lost to follow-up for unclear reasons. We cannot assume that more complete neurocognitive and psychiatric assessments were conducted for patients who did not show a favorable evolution as this may also reflect different practice among neurologists and/or a greater interest from some physicians towards these symptoms. Followup evaluation was available in only two cases, making definitive conclusion on longitudinal evolution impossible. At the same time, we feel this is an important issue to report. Patients with neurosyphilis should be followed longitudinally with general neurological assessments, neuropsychological testing, and psychiatric consultations at least at initial presentation and at six months post-treatment. This would help identifying residual deficits and functional impact, and help direct them to proper rehabilitation resources. We therefore recommend formal neurological, cognitive, psychiatric and functional assessment at baseline and six months after treatment for all patients with neurosyphilis.

\section{CONCLusion}

In recent years, the prevalence of neurosyphilis has risen again due to an increasing incidence of syphilis. Given its recrudescence, it is important to evoke the diagnosis when faced with atypical dementia or new-onset of a psychiatric disorder, especially in an older patient. Accurate diagnosis remains problematic, as the signs are not pathognomonic and the specificity and sensitivity of supportive laboratory tests are unclear. In fact, there is no gold standard for the diagnosis of neurosyphilis which often results in undue delays and serious consequences. Despite the fact that it is considered a reversible form of dementia, limited data on its long-term evolution exist in literature. Our results highlight this situation and further demonstrate the lack of rigorous follow-up in cognitive and psychiatric disturbances associated with neurosyphilis. Further efforts should include systematic longitudinal cognitive and psychiatric follow-up in neurosyphilis.

\section{ETHICS}

This study was approved by our local Research Ethics Board. 


\section{REFERENCES}

1. French P. Syphilis. BMJ. 2007;334(7585):143-7.

2. Musher DM. Syphilis, neurosyphilis, penicillin, and AIDS. J Infect Dis. 1991;163(6):1201-6.

3. Workowski KA, Berman S. Centers for Disease Control and Prevention. Sexually Transmitted Diseases Treatment Guidelines, 2010. MMWR Recomm Rep. 2010 Dec 17;59(RR12):1-110.

4. Ministère de la Santé et des Services Sociaux (MSSS). Syphilis infectueuse au Québec. Appel à la vigilance. Québec, Qué: MSSS; 2010.

5. Clark EG, Danbolt N. The Oslo study of the natural history of untreated syphilis; an epidemiologic investigation based on a restudy of the Boeck-Bruusgaard material; a review and appraisal. J Chronic Dis. 1955;2(3):311-44.

6. Rosahn PD. U.S.Federal Security Agency. Public Health Service. Autopsy studies in syphilis. 1947;suppl 21.

7. Merritt HH, Adams RD, Solomon HC. Neurosyphilis. New York: Oxford University Press; 1946.

8. Marra CM. Update on neurosyphilis. Curr Infect Dis Rep. 2009;11 (2):127-34.

9. Lukehart SA, Hook EW, 3rd, Baker-Zander SA, Collier AC, Critchlow CW, Handsfield HH. Invasion of the central nervous system by Treponema pallidum: implications for diagnosis and treatment. Ann Intern Med. 1988;109(11):855-62.

10. Guler E, Leyhe T. A late form of neurosyphilis manifesting with psychotic symptoms in old age and good response to ceftriaxone therapy. Int Psychogeriatr. 2011;23(4):666-9.
11. Wharton M, Chorba TL, Vogt RL, Morse DL, Buehler JW. Case definitions for public health surveillance. MMWR Recomm Rep. 1990;39(RR-13): 1-43.

12. Rosenbaum M. Similarities of psychiatric disorders of AIDS and syphilis: history repeats itself. Bull Menninger Clin. 1994;58(3): 375-82.

13. Zheng D, Zhou D, Zhao Z, et al. The clinical presentation and imaging manifestation of psychosis and dementia in general paresis: a retrospective study of 116 cases. J Neuropsychiatry Clin Neurosci. 2011;23(3):300-7.

14. Timmermans M, Carr J. Neurosyphilis in the modern era. J Neurol Neurosurg Psychiatry. 2004;75(12):1727-30.

15. Wilner E, Brody JA. Prognosis of general paresis after treatment. Lancet. 1968;2(7583):1370-1.

16. Brightbill TC, Ihmeidan IH, Post MJ, Berger JR, Katz DA. Neurosyphilis in HIV-positive and HIV-negative patients: neuroimaging findings. AJNR Am J Neuroradiol. 1995;16(4): 703-11.

17. Hama K, Ishiguchi H, Tuji T, Miwa H, Kondo T. Neurosyphilis with mesiotemporal magnetic resonance imaging abnormalities. Intern Med. 2008;47(20):1813-17.

18. Jeong YM, Hwang HY, Kim HS. MRI of neurosyphilis presenting as mesiotemporal abnormalities: a case report. Korean J Radiol. 2009;10(3):310-12.

19. Kodama K, Okada S, Komatsu N, et al. Relationship between MRI findings and prognosis for patients with general paresis. J Neuropsychiatry Clin Neurosci. 2000;12(2):246-50. 